\title{
Variaciones en el tratamiento de las sibilantes Inconsistencia en el seseo sevillano: Un enfoque sociolingüístico
}

\author{
Manuela González-Bueno \\ Pennsylvania State University
}

\begin{abstract}
El artículo de John B. Dalbor, "Observations on Present-Day seseo and ceceo in Southern Spain”, publicado en el $n^{\circ} 63$ de Hispania (1980), observa-efectivamente-y analiza el uso indiscriminado de los fonemas [s] y $[\theta]$ por parte de hablantes sevillanos, que no se atienen a una norma seseante ni ceceante de forma consistente. Esta indiscriminación lleva al autor a sospechar que ambos fonemas se encuentran "en variación libre." El propósito de este artículo es presentar una visión diferente del fenómeno, desde el punto de vista del nativo, y el aducir razones de índole sociolinguística para justificar la aparente confusión que la situación presenta-confusión generalmente en opinión de investigadores que, si bien poseen un extenso conocimiento lingüístico del fenómeno, carecen de la perspectiva sociolingüística que proporciona la experiencia del nativo.
\end{abstract}

Key words: seseo inconsistente, fonética (española), dialectos (España), Andalucía, Sevilla

M ucho se ha escrito y discutido a propósitodel seseo yceceo andaluces. Este fenómeno ha sido durante mucho tiempo motivo de numerosas investigaciones y polémicas entre lingüistas de reconocida importancia. El propósito de este trabajo no es rebatir ni tampoco repetir lo que otros linguiistas han aportado a este terreno, sino apoyándome en sus estudios y conclusiones, y contrastando éstas con las mías propias-que, como nativa de Sevilla, pueden aportar una luz nada desechable-contribuir a un mejor conocimiento de este fenómeno que nos ocupa.

En primer lugar, y por motivos de claridad, se impone una definición de estos dos conceptos-seseo y ceceo-, y para ello la tomaré prestada de John Dalbor: 'Supposedly 'seseo' speakers do not pronounce the interdental slit fricative phoneme $/ \theta /$ found in the national standard dialect 'castellano,' and 'ceceo' speakers do not pronounce its alveolar groove fricative phoneme /s/. Thus, where 'castellano' speakers contrast these two sounds for the letters $\mathbf{z}, \mathbf{c}^{+e, i}$ and $\mathbf{s}$, respectively, in all positions, as in cierra/sierra, caza/casa, Velázquez/Velasco and vez/ves, 'seseo' speakers pronounce all these words with /s/ and 'ceceo' speakers with $/ \theta /$ " (5).

Así pues, y tal como se desprende de esta definición, la distinción es la ausencia de es- tos fenómenos, es decir, la realización del contraste que se da en castellano entre estos dos sonidos. Este último comportamiento no es propio del habla andaluza en general, ni por tanto de la de Sevilla. Sin embargo, numerosas investigaciones, y una simple observación más o menos superficial, demuestran que es bastante común encontrar esta distinción en hablantes sevillanos. Como apunta Pedro Carbonero: "En lo referente a este rasgo la situación, desde luego, no es uniforme. Es posible encontrar casos de la distinción castellana, y si no hay distinción puede manifestarse como seseo, como ceceo, o incluso casos mixtos de hablantes seseantes o ceceantes que a veces distinguen y otras veces no. De cualquier manera, la solución generalizada en el ámbito urbano de Sevilla es el seseo: no distinción con manifiesta fonética seseante" ("Norma" 142).

Este fenómeno que tratamos es amplio y complejo y se puede estudiar desde diferentes puntos de vista. Existen razones históricas que explican esta doble situación seseante y ceceante en toda Andalucía. Dichas razones no solamente aportan los datos fonológicos sino también los criterios para la distribución geográfica de ambos fenómenos. Carbonero añade en otro trabajo: "La ciudad de Sevilla es un 'islote' de seseo en medio de toda una zona ceceante, aunque sin duda podrían encontrar- 
se en la ciudad algunos grupos de hablantes que cecean, sobre todo procedentes de otras localidades de la provincia y que han llegado a residir en la capital" (El habla.26).

Esta última observación es importante tenerla en cuenta pues, efectivamente, y como Sawoff ha observado también (238-62), estas migraciones han ocasionado que en ese "islote" se encuentren entremezclados hablantes seseantes y ceceantes.

Hasta ahora la situación no parece complicada, y los estudios estructuralistas que han tratado el tema del seseo y del ceceo así la han tomado. Pero esta situación se complica cuando se observa que un mismo hablante puede presentar los dos fenómenos de seseo y ceceo al mismo tiempo, y sobre todo que esta vacilación no parece responder a una regla fija. Así, por ejemplo-y ésta es una de las citas preferidas de Dalbor-podemos encontrar individuos que dicen: "El tren de las on[s] ey $[\theta]$ inco sale a las on[s]e y [ $\theta$ ]inco" (13).

Hechos de este tipo han llevado a los estudiosos a considerar ambos fonemas /s/ y $/ \theta /$ como extremos de un continuo que constituye un solo fonema, y que se representaría [s... $\theta$ ], explicando este hecho a través del concepto de "desfonologización." Carbonero habla de ésta como de un hecho diacrónico en que se da una mutación fonológica consistente en la desaparición de una oposición que existía en un momento diacrónico anterior y que deja de tener pertinencia en el sistema ("Polimorfismo" 52).

Esta teoría, que parece simplificar el panorama, hay que tomarla con mucha cautela, pues sería muy fácil caer en el error de pensar que un mismo hablante puede ir libremente de un extremo a otro de ese continuo, produciendo esa situación caótica ya apuntada por muchos.

Dejemos bien claro que hay tres tipos de hablantes en Sevilla: 1) el hablante seseante, en quien la desfonologicación ha ocurrido en favor de [s]; 2) el hablante ceceante, que se ha decantado por la realización $[\theta]$; y, por último, 3) el hablante, ya sea seseante, ya ceceante, que, por diferentes motivos que trataremos más tarde, realiza a veces una especie de "refonologización"-por lo demás antieconómica y arcaizante-haciendo la dis- tinción castellana entre $/ \mathrm{s} / \mathrm{y} / \theta /$.

Es este tercer tipo de hablante el que merece nuestra mayor atención. Para el primer tipo, el seseante, su solución a la falta de contraste $/ \mathrm{s} /-/ \theta /$ se situaría en el extremo [s] del continuo representado por [s... $\theta$ ] (representación, por lo demás, abstracta y que se adopta por razones metodológicas); el segundo tipo se situaría en el extremo [ $\theta]$. Pero es el tercer tipo el que contribuye a dar ese aspecto caótico a la pronunciación de las sibilantes en Sevilla, llevando a Sawoff a afirmar que la situación es un "confusing mixture of seseo, ceceo and SSD-Standard Spanish Distinction. It is, like seseo and ceceo, the phoneme reduction of Standard Spanish /s/ and $/ \theta /$, being the two allophones in free variation, also however, admitting an indeterminate number of intermediate articulations/allophones like [ $\left.{ }_{\theta}^{s}\right]$ " (241).

De esta afirmación pienso que habría que discutir lo de "confusing mixture" porque, en principio, no se da mezcla de seseo y ceceo, aunque si de seseo y distinción, así como también de ceceo y distinción. Esta mezcla no debe considerarse como confusa, ni tampoco que en ella los dos alófonos [s] y [ $\theta$ ] estén en variación libre totalmente. A esta idea volveré más adelante, cuando tratemos las posibles causas para esta "no-tan-libre" variacion.

Una de las causas que lleva a Sawoff a considerar como confusa esa mezcla es el propio método de investigación que utiliza, el cual él mismo reconoce en su artículo que tiene "some shortcomings" (242). El hecho de que un mismo hablante pronuncie la frase "ciruelas y manzanas" como " $[\theta]$ iruelas y man[s]anas" no significa que estè confundiendo seseo y ceceo, sino que está mezclando seseo ("man[s]anas") conSSD ("[ $\theta$ ]iruelas"), ya que esta última sería la pronunciación castellana. Igualmente podríamos decir que el que pronunciara “[ $\theta$ ]iruelas y man $[\theta]$ anas" puede ser ceceante o que simplemente está haciendo la distinción (repito que Sawoff reconoce estos defectos en su método, pero aun así ello no le impide insistir en calificar la situación como "confusa").

Bien es cierto que se pueden encontrary Dalbor lo hace-casos seseantes con desvío a ceceo, así como ceceantes con desvío a 
seseo, pero estos casos pueden explicarse por otros motivos. La simple explicación de que el hablante está balanceándose entre los dos extremos de [s...t] en variación libre no se corresponde con la realidad.

Sería más acertado interpretar que para cada uno de estos dos tipos de hablantes hay un sistema diferente, más o menos consistente, en el que los dos fonemas $/ \mathrm{s} / \mathrm{y} / \theta / \mathrm{del}$ castellano se han reducido a uno solo, /s/ para los seseantes, $/ \theta /$ para los ceceantes; dicho sistema se rompe cuando hay desvío, pero sólo lo hace por el lado del fonema que no fue favorecido en la reducción. Es decir, para el seseante, /s/ sigue siendo [s], y / $\theta$ / es a veces $[\mathrm{s}]-\mathrm{sese}-\mathrm{y}$ a veces $[\theta]$-distinción; mientras que para el ceceante, $/ \theta /$ sigue siendo $[\theta]$, pero $/ \mathrm{s} /$ puede ser ahora $[\theta]-$ ceceo-o [s]-distinción:

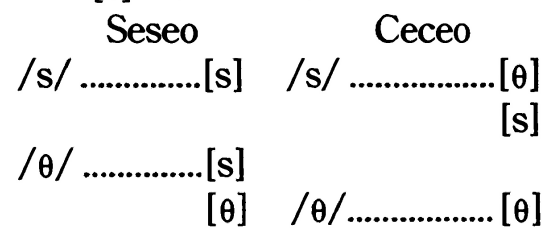

Según este esquema, un hablante seseante pronunciará la palabra "Zaragoza" como [s]arago[s]a o [ $\theta$ ]arago[ $\theta$ ]a, pero nunca pronunciará "Sevilla" como [ $\theta$ ] evilla (esto sería ceceo); mientras que un hablante ceceante podrá decir [ $\theta$ ] evilla o [s] evilla, pero nunca dirá [s]arago[s]a.

Esta situación así descrita es contradicha por Dalbor cuando afirma haber encontrado hablantes que:junto a un seseo bastante regular han demostrado desvio a SSD, pero también, y en el curso de la misma intervención, desvío a ceceo (13). Sin embargo, como nativa de Sevilla, mi percepción de la situación es la que acabo de explicar, y también, por el conocimiento directo del tema que me da tal condición, voy a intentar explicar estos desvíos que, como tales, no encajan como generalidades en el sistema que acabo de describir, sino como resultados ocasionales de ciertas causas sociolingüísticas que paso a analizar a continuación.

La principal causa que existe para explicar el desvío de los hablantes seseantes y ceceantes a la distinción es la presión de la norma oficial, y así es reconocido por todos los estudiosos que se han acercado mínimamente al tema. No se trata, sin embargo, de un disimular el acento andaluz-puesto que éste no consiste solamente en este rasgo-ni tampoco en demostrar que se es capaz de hacer la distinción (si asi fuera, las intervenciones linguiísticas de estos hablantes se convertirian en un juego de habilidades que dejarían de lado el principal objetivo de la comunicación).

Sería interminable hacer mención de todas las citas en las que estos estudiosos han apuntado estas causas. Sirvan de ejemplo algunas de ellas, pertenecientes a profesores y lingiiistas andaluces que en época reciente se han referido al tema.

El profesor de sociolingüística de la Universidad de Sevilla, Miguel Ropero, en su artículo "Identidad sociolingüística del andaluz," dice: “...lo normal es que los hablantes andaluces tengan cierto complejo de inferioridad y piensen que en Andalucía se habla mal (34)." Y más adelante agrega: "Son muchas y muy diversas (histórico-políticas, socioeconómicas, culturales, etc.) las causas que han originado en los hablantes andaluces [esa] valoración negativa de los propios usos lingüísticos. Una de estas causas, muy importante, a mi entender, es el tratamiento que los rasgos dialectales andaluces reciben en los libros de texto de EGB (Enseñanza General Básica, equivalente a la enseñanza primaria) y BUP (Bachiller Unificado Polivalente, equivalente a la enseñanza secundaria). A causa de [esta] valoración [...], la conciencia linguiística de los hablantes andaluces se debate en la actualidad entre el complejo de inferioridad y la aceptación, con cierto orgullo, de la propia modalidad linguística" (42).

No es de extrañar, pues, que los hablantes andaluces, llegado el momento de intervenir en situaciones más o menos formales, se desvien hacia una distinción aprendida en el colegio, hecho que me lleva a pensar en la posibilidad de una adquisición semi-inconsciente de ambos sistemas, el seseante o ceceante, según los casos, para situaciones coloquiales, y el distinguidor para situaciones más formales. Viene al caso citar en este punto al profesory lingüista andaluz José María Vaz de Soto, cuando dice:"El pueblo [andaluz] sabe que su modo de hablar está tan prohibido y menospreciado, que ni los mismos andaluces nos 
acogemos a él cuando se trata de hacernos oír en público o ante un micrófono (19).

Para ilustrar este "poder" del micrófono, sirva el ejemplo del locutor de radio Matías Pratt, natural de Córdoba-zona seseantey comentarista de toros y de fútbol ya retirado, que ante la dificultad que encontrara para pronunciar el sonido / $\theta$ / opuesto a / s/ - distinción obligatoria impuesta por el ente radiofónico de la época para poder ejercer como locutor-prefirió hacer el esfuerzo de sustituirlo por el sonido /f/, de articulación fricativa cercana, con tal éxito que hoy en día, y aunque ya parece que "se levantó la veda" para las pronunciaciones dialectales en entes públicos como Radio-Televisión Española, todavía mantiene esa costumbre el citado senor, y a no ser que se le preste mucha atención, es dificil darse cuenta de que frases como "favorecer la situación" él la pronuncia como "favore[f]er la situa[f]ión."

De mi experiencia personal debo decir que, si bien recuerdo a casi todos mis profesores como no andaluces, nunca tuve conciencia de que se me corrigiera en ningún momento mi pronunciación seseante (de ahí mi idea de la adquisición "semi-inconsciente" de ambos sistemas.) No obstante, ya en cursos superiores de la Universidad, se me pidió en cierta ocasión que leyera en voz alta, y desde el entarimado, un poema de García Lorca que estaba escrito en la pizarra. A pesar de tratarse de una situación formal, la clase de Critica Literaria, y de estar dirigiéndome a un público, mis compañeros de clase, parece que me sentí lo suficientemente relajada como para leer el poema entero con un seseo recalcitrante que hubiera molestado al propio Garcia Lorca si me hubiera oído (aunque estoy segura de que él habría sido más tolerante, como andaluz que era). Al terminar, mi profesorandaluz también-me hizo repetir la lectura, esta vez "leyendo y pronunciando lo que pone ahi"-fueron sus palabras-es decir, haciendo la distinción que la formalidad de la circunstancia requería.

Tal vez sea relativamente fácil para un andaluz culto (un profesor, un político, un locutor...) mantener cierta consistencia en la distinción cuando habla en público o ante un micrófono, pero no todo el mundo que se si- túa detrás de uno ha tenido la oportunidad de adquirir ese otro "sistema de repuesto" que todos los andaluces parecemos obligados a utilizar llegado el momento. Es entonces cuando, de manera artificiosa se adopta dicho sistema, sin mucho éxito la mayoría de las veces, dándose asi casos de ultracorrección, o simplemente confusión. Y es en estas situaciones donde opino que encajarían los casos de desvio a ceceo a que Dalbor se refiere en su artículo. No son, pues, actuaciones ceceantes como tales los casos de "a[ $[\theta] i$, " "[ $[\theta]$ eñor" o "[ $\theta$ ]emana" cuando son pronunciados por hablantes que han demostrado un seseo más o menos consistente, sino casos de ultracorrección ocasionados por la artificiosidad o formalidad de la situación.

Otras veces, los casos de inconsistencia se complican cuando provienen de hablantes que han sido anteriormente ceceantes. Estos casos no son extraños. Hay que tener en cuenta que, dentro de la pronunciación andaluza, la seseante goza de mayor prestigio social que la ceceante, lo cual provoca que, despues de un tiempo, los hablantes ceceantes que se han trasladado a Sevilla acaben por adoptar el seseo, aunque, como es de esperar, con ocasionales desvíos al ceceo original. En este sentido, Carbonero apunta: “...la pronunciación seseante es considerada socialmente más culta y refinada, mientras que la ceceante se ve como más rústica y vulgar. Aunque desde una perspectiva linguística no hay razones para preferir un uso u otro, no hay duda de que se trata de un fenómeno de prestigio social" (EI habla 27).

Un caso cercano a mi experiencia es el de mi propia madre, que, procedente de una zona ceceante-Aznalcóllar, localidad a $60 \mathrm{~km}$. de Sevilla-dejó su ceceo cuando se desplazó a Sevilla en su adolescencia, para adoptar un seseo en general bastante consistente, que abandonaba solamente cuando se refería a personas o cosas relacionadas con su infancia ceceante. Así, por ejemplo, cuando nombraba a su amiga del colegio Josefa, este nombre era pronunciado como "Jo[ $\theta]$ efa."

Igual inconsistencia se observa en hablantes andaluces tan populares como Lola Flores o Rocío Jurado, ambas cantantes folclóricas y procedentes de zonas ce- 
ceantes-Jerezy Chipiona, respectivamente, de la provincia de Cádiz; tales personajes, a fuerza de estar sometidos a situaciones formales (entrevistas en televisión, radio, prensa...), unido a que, debido a su profesión, cambiaron hace tiempo su residencia a Madrid, junto con la exigencia de mantener su acento andaluz como estandarte de su arte, denotan una inconsistencia extrema en el tratamiento de las sibilantes. Es el caso de la primera - $\mathrm{La}$ Lola"_el más pintoresco, pues ella puede, en el curso de una misma frase, hacer distinción, sesear y dar ejemplo de su ceceo jerezano. Es el caso de la siguiente intervención, tomada de una entrevista recientemente llevada a cabo en la televisión española: "Y [s] oy diva cuando tocan el timbre y [s]uena la mú[s]ica [...], después [s] oy una per[ $\theta$ ]ona bastante [s]en[s]illa. [...] Y creo que Je[s]ús le da a cada uno lo que [s]e mere[ $\theta$ ]e, pero el daño que me hi[s]ieron de tirar mi nombre por el [s]uelo [...] no lo olvidaré jamás. [...] Estoy viendo muchas co[s]as que a este pa[ $\theta]$ o soy [s]anta Tere[s]a de Je[s]ús."

Este tipo de situaciones añade gran inconsistencia y aparente confusión al observador externo. A este respecto, Vaz de Soto apunta: "No estará de más advertir que, en muchos hablantes cultos, sobre todo de origen ceceante, y sin duda por presión de la norma oficial, se observa hoy una clara vacilación entre el seseo y la distinción más 0 menos rigurosa de los dos fonemas $(/ \mathrm{s} /-/ \theta /)$ según la norma de Castilla" (19).

Parece ser, pues, que estos hablantes ceceantes, al haber tenido que pasar por dos "filtros," el del seseo primero, el de la distinción después, son los que mayor índice de vacilación aportan a todo este "maremágnum" de sibilantes.

Espero que las razones aquí aducidas para explicar estas vacilaciones sean suficientes para desterrar la idea de que la libre variación determina la elección de uno $\mathrm{u}$ otro fonema. Cuando un hablante elige usar uno de ellos, tiene detrás de esa elección varias razones para hacerlo. Puede que para un hablante seseante el contraste $/ \mathrm{s} /-/ \theta /$ ya no funcione a nivel fonológico, pero si ocasionalmente alguna vez elige usarlo, es decir, decide hacer la distinción, no es porque la posibilidad $[\theta]$ se le ofrezca como una alternativa más, en libre variación, sino que hay una razón, tal vez no puramente lingüistica, que le empuja a ello. Al fin y al cabo estamos tratando con sistemas, y como nos hacer recordar Labov, "a system is essentially a set of differences" (5). Y continúa más adelante: "The complexities found in the exploratory interviews may appear to justify the view that New York City speech is chaotic, and that 'free variation' is indeed an adequate description. But free variation on a scale such as this is hardly consonant with the concept of a coherent, interrelated system" (31).

En su impresionante estudio sobre la estratificación sociolinguíística del inglés en la ciudad de Nueva York, Labov desarrolla un método de investigación y llega a unas conclusiones que muy bien podrían aplicarse al tema que analizamos. Sobre todo su idea sobre la no operatividad de la variación libre al tratar todos estos elementos variables de la lengua. De hecho, él intenta demostrar cómo todos ellos están determinados de una forma o de otra: "This attitude is grounded in the conviction that language is no less determinate than other forms of social behavior" (32).

Me aventuraría aquí a rastrear otras posibles causas, más relacionadas con el contexto fonético, para algunos casos de inconsistencia en el uso de un sistema u otro, es decir, en casos de hablantes cultos que, aunque capaces de permanecer de manera consistente en un sistema seseante o ceceante, $o$ elegir el sistema distinguidor, presentan, en situaciones semi-formales, ciertas inconsistencias. Ya Dalbor parece encontrar ciertos patrones de asimilaciones (13). Pienso que sería muy difícil explicar estas inconsistencias o desvíos por medio de este fenómeno, aunque a veces lo he considerado. Pero, tal como Dalbor reconoce, estos casos no supondrían más que ocasionales desvíos, no significativos dentro de la consideración más amplia que el fenómeno que nos ocupa merece.

Sí me parecería más defendible la teoría de que el hecho de que una palabra sea más o menos coloquialy, por tanto, el hablante esté más acostumbrado a utilizarla, influya en la manera de pronunciar la misma. Esta idea la 
sugiere de nuevo Dalbor cuando, después de aportar los ejemplos de las palabras "haciendo" y "Preciados" como casos de desvío a seseo dentro de una intervención que hasta ese momento había sido consistentemente distinguidora, añade: “...both, of course, extremely common words in Spain (12). Para mí, éste es un caso de contaminación de los dos sistemas, seseante y distinguidor, detrás del cual volvemos a ver causas sociolingüísticas.

Observándome a mi misma, me veo-me oigo, debería decir-pronunciando la palabra gracias con [ $\theta$ ] la mayoría de las veces, y con menor frecuencia con [s]; aunque gracias es una palabra muy corriente en español, también es cierto que es una fórmula de cortesía, que en la mayor parte de los casos se pronuncia en situaciones formales. Si pienso en la palabra civilización, afirmaría que la pronuncio con todas sus ces y sus zetas: “ $[\theta]$ ivili $[\theta] a[\theta]$ ión," y ello no se debería a otra cosa que a la formalidad del contexto en que dicho término sería usado. Lo cual no quiere decir que en un contexto menos formal no volviera a mi seseo habitual para pronunciar "[s]ivili[s]a[s]ión." En cualquiera de los casos, habría una elección por parte del hablante, basada en cuestiones sociales, que invalida la teoría de que ambas realizaciones, $[\mathrm{s}]$ y $[\theta]$ se encuentran en variación libre.

Todo esto está en consonancia con las conclusiones a que llega Labov en su estudio sobre las variantes estilísticas y sociales en el inglés de Nueva York. Él afirma: "Further explorations of New York City speech revealed more of the pattern behind this variation" (37).

La mala conciencia que en general ha habido entre los andaluces sobre nuestra manera de pronunciar las sibilantes se ha perpetuado por siglos, y probablemente siga ocurriendo así durante mucho tiempo, lo cual seguirá debiéndose a causas de prestigio social, situación que, si bien tolerada y admitida por los propios andaluces, tiene su origen, como es natural, en la consideración externa de hablantes españoles de otras regiones. Pero esta situación es susceptible de cambio, pues, como Carbonero afirma: “...la consideración de la norma estándar se sostiene sobre las creencias y la 'conciencia' sociolingüistica de los hablantes de la comunidad en cuestión, que tienen esa norma como modelo a seguir, por razones de prestigio social. Y debido a ello, puede ocurrir que la conciencia social-por razones histórícas, políticas y culturales muy diversas-cambie, a veces, con relativa rapidez. Lo que en un momento histórico puede estar bien considerado, puede pasar a no estarlo tanto, y viceversa, según los avatares de la evolución sociocultural de la sociedad" ("Norma" 138-39).

Si en un pasado no remoto, cuando el "telespañolito" enchufaba la tele, los únicos seseos y ceceos a que tenía acceso eran los de los personajes de los sainetes de los Álvarez Quintero, quienes con sus “ozuis” y sus "olé la grasia" divertían al personal, ahora tienen también la oportunidad de escuchar seseos en boca de una elegante, seria y culta presentadora canaria, o de una inteligente y dinámica reportera en Moscú, e incluso de los mismísimos Presidente y ex-Vicepresidente del gobierno, Felipe González (léase "Gon[s]ále[s]") y Alfonso Guerra, ambos de Sevilla. ¡Y hasta ceceo se escucha, cuando aparece entrevistado el Sr. Lara, presidente de la Editorial Planeta y patronizador del Premio Planeta, uno de los más prestigiosos premios literarios en lengua castellana!

No creo en absoluto que exista una tendencia, ni en Sevilla ni espero que en ningún otro sitio de Andalucia, hacia la distinción castellana, como Sawoff quiere demostrar. Como queda ya dicho más arriba, sería un caso de involucionismo arcaizante y antieconómico. Tampoco que el seseo, junto con los otros rasgos andaluces, vaya a extenderse hasta la cornisa cantábrica, como tan triunfalmente profetiza Gregorio Salvador (188).

1 reajuste fonológico de las sibilantes que se dio en castellano en los siglos XV y XVI fue el principio de una situación de tensiones que, aparentemente, no llegaron a resolverse en Andalucia. Esta situación, como es evidente, aún continúa, y existen tendencias niveladoras hacia una $u$ otra dirección, que no parecen conciliarse en una solución única. Lo cual me parece excelente. Me gusta la idea de un hablante andaluz, sevillano versátil, diferente de los otros, tolerante 
y tolerado, con la capacidad de elegir según las circunstancias. No abogo por la idea de considerar la pronunciación andaluza como confusa, inconsistente, simuladora 0 pretenciosa. Reconozco que al observador externo-extranjero, extraño-toda esta situación le pueda parecer confusa. Pero los sevillanos tenemos nuestras razones.

\section{- OBRAS CITADAS}

Carbonero Cano, Pedro. "Norma estándar y actitud sociolingüistica." Sociolingüústica Andaluza I. Sevilla: Departamento de Lengua Española, Universidad de Sevilla, 1982.

. "Polimorfismo y funcionalidad en el uso lingiüstico de hablantes andaluces." Sociolingüística Andaluza I. Sevilla: Departamento de Lengua Española, Universidad de Sevilla, 1982.
El habla de Sevilla. Sevilla: Servicio de Publicaciones del Ayuntamiento de Sevilla, 1982.

Dalbor, John B. "Observations on Present-Day seseo and ceceo in Southern Spain." Hispania 63 (1980): 5-19.

Labov, W. The Social Stratification of English in New York City. Washington D.C.: Center for Applied linguistics, 1982.

Ropero, Miguel. "Identidad sociolinguística del andaluz." Sociolingüústica Andaluza I. Sevilla: Departamento de Lengua Española, Universidad de Sevilla, 1982.

Salvador, Gregorio. "La fonética andaluza y su propagación social y geográfica." Presente y futucro de la lengua española I. Madrid: Cultura Hispánica, 1964.

Sawoff, A. "A sociolinguistic Appraisal of the Sibilant Pronunciation in the City of Seville," Grazar Linguistische Studien, 11/12. Festgabe für Norman Denison, Frühjahr (1980).

Vaz de Soto, Jose M. Defensa del habla andaluza. Sevilla: Consejería de Cultura de la Junta de Andalucía, 1981. 